\title{
Salud y acceso a cuidados médicos durante la pandemia en Uruguay
}

Health and access to medical care during the covid-19 pandemic Saúde e acesso a cuidados médicos durante a pandemia no Uruguai

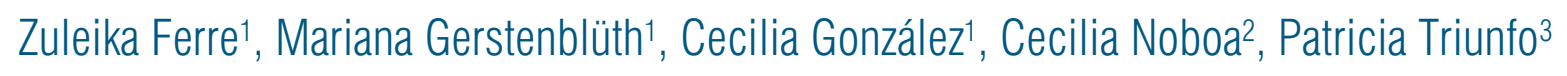

\section{Resumen}

Introducción: la pandemia provocada por la COVID-19 ha cambiado drásticamente las modalidades de atención médica ambulatoria y cuidado de la salud. Uruguay implementó, desde el 15 de marzo de 2020, una serie de cambios en el sistema de salud para focalizar los recursos disponibles y evitar contagios.

Objetivo: presentar los resultados de la Encuesta sobre Salud y Acceso a Cuidados Médicos (ESAC) durante la emergencia sanitaria originada por el COVID-19 en Uruguay en el período desde el 13 de marzo al 30 de mayo de 2020. El objetivo es mostrar el impacto que la pandemia tuvo sobre algunos aspectos de la atención médica recibida.

Material y métodos: la ESAC, dirigida a residentes en Uruguay mayores de 18 años, fue aplicada a través de un cuestionario autoadministrado por internet entre el 10 y el 27 de julio de 2020. La muestra total es de 1.750 encuestas y relevó información relativa a la salud y cuidados médicos.

Resultados: la crisis sanitaria propició un cambio en la modalidad de la práctica clínica, con una alta participación de la telemedicina, la cual fue mayoritariamente definida por el centro de salud. Se encontró una alta satisfacción en la gestión y atención durante la pandemia. Sin embargo, destaca el alto porcentaje de estudios médicos, procedimientos o tratamientos en curso o previstos que fueron suspendidos o pospuestos. En el caso de los niños menores de 12 años, se encuentra que más de la mitad tuvo que retrasar alguna vacuna prevista.

Conclusiones: en el futuro será necesario que el sistema de salud preste especial atención a la forma en que se definen e implementan las nuevas modalidades de consulta, tiempos de espera máximos, y la correcta atención a quienes padecen enfermedades crónicas, para garantizar el acceso a cuidados médicos oportunos y de calidad.

Palabras clave: COVID-19

Uruguay

Atención médica

Accesibilidad a los servicios de salud

Key words: $\quad$ COVID-19

Uruguay

Medical care

Health services accessibility

\footnotetext{
1. Profesora Adjunta. Departamento de Economía, Facultad de Ciencias Sociales, Universidad de la República

2. Asistente. Departamento de Economía, Facultad de Ciencias Sociales, Universidad de la República

3. Profesora Titular. Departamento de Economía, Facultad de Ciencias Sociales, Universidad de la República

Los autores declaran no tener conflictos de intereses.

Correspondencia: Mariana Gerstenblüth. Correo electrónico: mariana.gerstenbluth@cienciassociales.edu.uy

Recibido: 11/2/2021

Aprobado: 18/5/2021

Attribution-NonCommercial 4.0 International (CC BY-NC 4.0)
} 


\section{Introducción}

La pandemia provocada por la COVID-19 ha cambiado drásticamente las modalidades de atención médica ambulatoria y el cuidado de la salud. La alta velocidad de propagación de la enfermedad constatada al inicio de la pandemia, determinó que muchos países adoptaran como medidas la postergación de las visitas electivas y preventivas presenciales para evitar el contagio de pacientes y trabajadores del sector de la salud.

En Uruguay, el 15 de marzo la Dirección General de la Junta Nacional de Salud de Uruguay (JUNASA) tomó una serie de resoluciones que buscaron focalizar los recursos disponibles en la atención. En el comunicado se apelaba a la responsabilidad y solidaridad de la población, solicitando a los ciudadanos a minimizar la concurrencia a centros asistenciales, limitándola a situaciones de gravedad. A su vez, los prestadores de salud quedaron autorizados a dar prioridad a la atención domiciliaria y a la utilización de medios alternativos de atención (telemedicina) para control y seguimiento de patologías no graves. También se permitió la reprogramación de las intervenciones quirúrgicas de coordinación no urgentes, excluidas las vinculadas con patologías oncológicas. Finalmente, se propuso flexibilizar el contralor de las metas asistenciales establecidas por el Ministerio de Salud Pública (MSP), cuyo cumplimiento puede verse afectado por las anteriores medidas ${ }^{(1)}$. Por otro lado, en Uruguay no se aplicó un confinamiento obligatorio, a la vez que las primeras resoluciones se tomaron dos días después de detectarse los primeros cuatro casos ${ }^{(2)}$.

En este documento se presentan los resultados de la Encuesta sobre Salud y Acceso a Cuidados Médicos durante la pandemia en Uruguay (ESAC) realizada entre el 10 y el 27 de julio de 2020, que pretende recabar información relevante para monitorear problemas de acceso a la atención médica, los cuales pueden agravarse ante las estrategias de distanciamiento seguidas durante la crisis sanitaria. Comprender cómo las personas están experimentando este período es fundamental para dar respuestas gubernamentales y no gubernamentales de forma oportuna.

\section{Material y métodos}

La ESAC estuvo a cargo del Departamento de Economía de la Facultad de Ciencias Sociales de la UdelaR. La encuesta se realizó a través de un cuestionario autoadministrado por internet a mayores de 18 años residentes en Uruguay. Fue difundido a través de redes sociales, correo electrónico y sitio web de Facultad de Ciencias Sociales y estuvo disponible entre el 10 y el 27 de julio de 2020. La muestra total es de 1750 encuestas efectivas.
Se relevó información relativa a la salud y cuidados médicos durante la crisis sanitaria en Uruguay en el período del 13 de marzo al 30 de mayo de 2020. El foco estuvo en recoger información acerca de la atención médica (tanto en policlínica, emergencia móvil o en domicilio), así como la satisfacción con la misma. En particular, el interés estuvo en conocer las necesidades y modalidades en las que se concretaron las consultas médicas. Además, se relevó información sobre el estado de salud, preocupación respecto a la pandemia, hábitos alimentarios y de actividad física.

La utilización de internet tiene ventajas, sobre todo en lo referido a rapidez y bajo costo. Sin embargo, tiene como limitantes el problema del sesgo muestral. Para lidiar con este problema, para la ESAC se crearon ponderadores asociados con la probabilidad de selección del individuo en base a tres características: sexo, nivel educativo (primaria, secundaria y terciaria) y región de residencia (Montevideo o resto del país). El ponderador es igual al inverso de su probabilidad de inclusión, por lo tanto es igual al porcentaje del grupo $i$ definido por las variables mencionadas en el Censo de Población del año 2011 y el porcentaje de dicho grupo en la ESAC.

A efectos de comparar con datos recientes, en la tabla 1 se presenta la distribución de las principales variables sociodemográficas presentes tanto en la ESAC (datos ponderados) como en la Encuesta Continua de Hogares (ECH) 2019 (cuyo marco muestral es el Censo 2011).

La variable nivel educativo distingue tres niveles: Primaria, Secundaria y Terciaria. En cada uno de ellos se incluyen aquellas personas que declaran haber alcanzado dicho nivel, ya sea incompleto o completo, como el máximo nivel educativo.

La variable cobertura de salud incluye las siguientes categorías: Administración de los Servicios de Salud del Estado (ASSE) (quienes declaran tener exclusivamente cobertura pública), Instituciones de Asistencia Médica Colectiva (IAMC) (quienes declaran tener exclusivamente cobertura privada en IAMC), Seguro Privado (cobertura privada pero no en IAMC) y Otros (que incluye cobertura en el Hospital Policial, Hospital Militar, otras coberturas, y también a quienes declaran doble cobertura).

Dado que en la ESAC no se dispone del ingreso del hogar, se construyó un índice de privación que aproxima el nivel socioeconómico a través de la disponibilidad de bienes y servicios en el hogar (freezer, televisión por cable, acceso a plataforma de streaming, lavarropa, secarropa, lavavajilla, automóvil, cantidad de calefones, cantidad de computadoras, servicio doméstico y propiedad de la vivienda). En primer lugar, se construye un índice de bienes y servicios donde cada uno es ponderado por la ausencia relativa de dicho bien en el total de los 
Tabla 1. Distribución porcentual de sexo, edad, nivel educativo, región y cobertura de salud de los entrevistados de la ESAC-2020 y la ECH-2019.

\begin{tabular}{|c|c|c|c|c|c|c|}
\hline & \multicolumn{3}{|c|}{ ECH 2019} & \multicolumn{3}{|c|}{ ESAC } \\
\hline & Porcentaje & \multicolumn{2}{|c|}{ Intervalo de confianza (95\%) } & Porcentaje & \multicolumn{2}{|c|}{ Intervalo de confianza (95\%) } \\
\hline \multicolumn{7}{|l|}{ Sexo } \\
\hline Femenino & 52,6 & 52,5 & 52,6 & 49,1 & 39,6 & 58,5 \\
\hline Masculino & 47,4 & 47,4 & 47,5 & 50,9 & 41,5 & 60,4 \\
\hline \multicolumn{7}{|l|}{ Edad } \\
\hline 18 a 29 & 23,1 & 23,1 & 23,2 & 10,6 & 6,1 & 15,1 \\
\hline 30 a 44 & 26,6 & 26,6 & 26,7 & 19,1 & 14,4 & 23,9 \\
\hline 45 a 54 & 16,8 & 16,7 & 16,8 & 16,8 & 11,8 & 21,9 \\
\hline 55 a 64 & 14,3 & 14,3 & 14,4 & 30,3 & 20,6 & 40,1 \\
\hline 65 y más & 19,2 & 19,1 & 19,2 & 23,1 & 13,1 & 33,2 \\
\hline \multicolumn{7}{|l|}{ Nivel educativo } \\
\hline Primaria & 34,3 & 34,2 & 34,3 & 32,5 & 20,2 & 44,8 \\
\hline Secundaria & 48,8 & 48,7 & 48,9 & 48,2 & 38,9 & 57,5 \\
\hline Terciaria & 162 & 16,2 & 16,3 & 19,3 & 15,5 & 23,2 \\
\hline \multicolumn{7}{|l|}{ Región } \\
\hline Montevideo & 40,6 & 40,5 & 40,6 & 41,7 & 33,2 & 50,1 \\
\hline Interior del país & 59,4 & 59,4 & 59,5 & 58,3 & 49,9 & 66,8 \\
\hline \multicolumn{7}{|l|}{ Cobertura de salud } \\
\hline $\begin{array}{l}\text { Administración de los Servicios de Salud } \\
\text { del Estado }\end{array}$ & 31,0 & 30,9 & 31,0 & 17,1 & 11,5 & 22,3 \\
\hline Instituciones de Asistencia Médica Colectiva & 61,0 & 60,9 & 61,0 & 64,4 & 54,6 & 74,2 \\
\hline Seguros privados & 2,4 & 2,3 & 2,4 & 3,8 & 2,4 & 5,3 \\
\hline Otras & 9,1 & 9,1 & 9,2 & 10,2 & 0,0 & 20,8 \\
\hline Doble cobertura & 4,9 & 4,9 & 5,0 & 4,5 & 2,4 & 6,6 \\
\hline $\mathrm{N}$ & 2.644 .785 & & & 1.750 & & \\
\hline
\end{tabular}

hogares. En segundo lugar, se realiza la suma ponderada de todos los bienes y servicios del hogar, de tal forma que a menor cantidad de bienes en el hogar mayor es el valor del índice (en el extremo un hogar que no tiene ninguno de los bienes o servicios considerados tomará el valor 1). Finalmente, se construye el indicador de privación relativa que toma el valor 1 si el índice de disponibilidad de bienes y servicios del hogar es mayor que la mediana de la distribución ${ }^{(3)}$.

La variable estado de salud es una variable auto-reportada, que se obtiene de la siguiente pregunta: "En general, ¿usted diría que su estado de salud es: excelente, muy bueno, regular, bueno o malo?".

La variable condición médica diagnosticada se construye si el entrevistado declara tener al menos una de las siguientes condiciones diagnosticadas por un mé- dico: asma, EPOC, hipertensión, enfermedades cardíacas, diabetes, inmunodepresión, sobrepeso, depresión o trastornos de ansiedad.

\section{Resultados}

En el anexo 1 se presentan las tablas que recogen los principales resultados de la encuesta, con los respectivos intervalos de confianza. A continuación, se comentan únicamente los resultados estadísticamente significativos, en todos los casos se aplicaron pruebas de diferencia de medias para las aperturas consideradas.

\section{Acceso a consultas y estudios médicos}

La tabla A1 muestra los resultados a nivel general sobre la forma en que se llevaron a cabo las consultas médicas durante el período comprendido entre el 13 de marzo y 
el 30 de mayo. El 47\% del total de entrevistados tenía agendada o tuvo necesidad de tener una consulta médica durante el período mencionado. De ellos, el 34\% correspondía a una consulta en policlínica, mientras que el $15 \%$ era en domicilio (ya sea con especialista o médico de radio).

Entre quienes necesitaron consultas en policlínica, la amplia mayoría (77\%) pudo concretar al menos una consulta, ya sea de forma presencial (54\%) o de forma telefónica o a través de video llamadas (telemedicina) (73\%). Respecto a las consultas en domicilio, $91 \%$ pudo concretar al menos una, las que fueron mayoritariamente realizadas en la modalidad de telemedicina (79\%).

Centrándonos en las consultas en policlínica, se observa que las mujeres necesitaron una mayor cantidad de consultas que los hombres, así como las personas de mayor nivel educativo y los que pertenecen a hogares con menor privación relativa. Tal como era de esperar, el tener una condición médica diagnosticada implicó una mayor necesidad de consultas médicas, sin embargo no se encuentran diferencias significativas en cuanto al acceso. En cambio, sí se encuentran mayores problemas de acceso para las mujeres y los que tienen cobertura pública, en cualquier modalidad (tabla A2).

\section{Modalidad de prácticas clínicas}

Con motivo de la emergencia sanitaria muchas consultas fueron canceladas o reagendadas, a la vez que las que se realizaron tuvieron lugar en una modalidad distinta a lo habitual. De hecho, se observa que entre quienes tenían una consulta agendada o necesitaron consultas en policlínicas y pudieron realizar al menos una en el período, $59 \%$ de las consultas fue a través de telemedicina, $38 \%$ fue presencial y $3 \%$ en otras modalidades. A su vez, entre quienes tenían agendada o necesitaron consultas con médico de radio o especialista a domicilio, $72 \%$ de las consultas fue por telemedicina y el $27 \%$ fue de modo presencial (tabla A3).

Como se muestra en la tabla A4, respecto a quienes necesitaron consultas en policlínica y lograron tener al menos una, el $44 \%$ de los entrevistados no tuvo ninguna consulta presencial, el 32\% tuvo una combinación de modalidad presencial y telemedicina, y aproximadamente el $24 \%$ tuvo todas sus consultas de forma presencial. Al analizar por subgrupos, se encuentran diferencias significativas entre los menores de 30 años y los mayores, observándose la estrategia de disminuir la concurrencia a los centros de salud, ya que el 5\% de los de 65 años o más tuvo todas las consultas presenciales versus el $60 \%$ de los jóvenes. Se destaca, además, que los que tienen cobertura pública son los que en mayor medida tuvieron consultas presenciales en policlínica.
En la tabla A5 se presenta el porcentaje de consultas de médico de radio o especialista a domicilio por tipo de modalidad, observándose que el $60 \%$ de los entrevistados declaran no haber tenido ninguna presencial, $25 \%$ en modalidad combinada, y el $15 \%$ exclusivamente de forma presencial. Las diferencias observadas son significativas para diversos subgrupos, encontrándose menor presencialidad en los hombres, en los mayores de edad, en los menos educados, y en quienes tienen mayor privación relativa y que viven en el interior del país. En particular, respecto al sexo, hay mayor presencia de telemedicina en los hombres $(84 \%$ versus $27 \%$ de las mujeres), de hecho apenas 3,5\% declara haber tenido todas estas consultas de forma presencial, mientras que las mujeres casi en un $32 \%$. Por nivel educativo, declaran no haber tenido consultas presenciales en mayor medida los encuestados con menor nivel educativo ( $80 \%$ primaria, $37 \%$ secundaria y $32 \%$ terciaria). En cuanto a la región, parece haber habido un mayor cambio de modalidad en el interior del país, donde el 78\% declara no haber tenido este tipo de consultas de forma presencial, versus el 21\% en Montevideo. Por estado de salud, hay diferencias significativas para aquellos que declaran un estado malo o regular (79\% no tuvo ninguna consulta presencial), versus el resto ( $32 \%$ estado bueno y $36 \%$ estado de salud muy bueno o excelente). Del mismo modo, los que tienen al menos una condición médica diagnosticada declaran en mayor medida no haber tenido este tipo de consultas de forma presencial (65\% versus $26 \%$ ).

Respecto a quién definió la modalidad de las consultas (tabla A6), se observa que para aquellas agendadas en policlínica, cuando se realizó en forma de telemedicina en $80 \%$ de los casos fue el centro de salud quien definió esta modalidad, mientras que correspondió al 53\% de los casos si se realizó en forma presencial. En el caso de consultas agendadas a domicilio, se observa que entre quienes tuvieron consultas presenciales $57 \%$ declara que fue el centro de salud, mientras que si fue por telemedicina, $72 \%$ manifiesta que lo decidió el entrevistado. Por lo tanto, se observa que en el caso de las consultas en policlínica fue el centro de salud el que tomó la decisión de la modalidad de telemedicina, mientras que en el caso de las consultas a domicilio, fueron los usuarios los que tomaron dicha decisión.

En línea con los resultados anteriores, interesa destacar que entre quienes tenían agendada una consulta en policlínica y la tuvieron de forma telefónica, 36\% manifiesta que hubiese preferido otra modalidad (tabla A7). Consultados sobre los motivos del cambio en la modalidad de la consulta, cancelación o cambio de fecha, el $50 \%$ de los entrevistados dice que fue el centro de salud que no le dio otra opción, y un 16\% aduce motivos de te- 
mor, ya sea de salir, de ingresar a un centro de salud o de usar el transporte público (tabla A8).

Respecto a aquellos entrevistados que tenían algún estudio médico, procedimiento o tratamiento en curso o previsto durante la pandemia (por ejemplo ecografías, tomografías, radiografías, papanicolau, mamografía, fisioterapia, colocación de DIU, cirugías, etc.), únicamente $11 \%$ pudo realizar todo lo que tenía previsto, destacándose que $72 \%$ de los entrevistados no pudo realizar ningún estudio en este período (tabla A9). Se encuentran diferencias significativas por cobertura y según estado de salud. Los usuarios de IAMC y seguros privados declaran en una mayor proporción haber podido realizar todos los estudios previstos, respecto a quienes tienen cobertura médica por ASSE (30\% seguro privado, 14\% IAMC y $6 \%$ de ASSE).

En términos generales, $68 \%$ de los entrevistados dice estar de acuerdo o muy de acuerdo con la modalidad en la que tuvo la o las consultas, a la vez que $78 \%$, indica que la atención médica estuvo de acuerdo con sus expectativas, y $72 \%$ manifiesta que el centro de salud gestionó correctamente los cambios e imprevistos surgidos durante la crisis sanitaria (tabla A10). Sin embargo, se encuentran diferencias en la satisfacción con la atención médica recibida entre quienes tienen educación primaria y terciaria, siendo menor para estos últimos. En cuanto a la gestión, están más satisfechos los del interior del país, los que tienen mayor privación relativa, y los que tienen cobertura a través de ASSE o seguros privados en relación a IAMCs.

\section{Acceso a cuidado médico por grupo etario}

La ESAC incluyó un bloque referido a los cuidados médicos de los menores de 12 años. En el mismo se preguntó a cada entrevistado/a con hijos en ese tramo, que responda respecto al hijo/a de menor edad. El $52 \%$ de los niños necesitó una consulta pediátrica durante la emergencia sanitaria, de los cuales el $70 \%$ accedió (tabla A11). La necesidad de consultas pediátricas fue más alta para los niños de hasta 2 años respecto al resto. También se encuentran diferencias significativas en el acceso a dichas consultas, con porcentajes mayores para los niños de hasta 5 años, respecto a los mayores de 6 (entre 80 y $85 \%$ versus $57 \%$ ). Este resultado va en línea con el hecho que la frecuencia de controles pediátricos requerido para los menores de 3 años es mayor que para el resto. En lo que respecta a la modalidad, mientras que el $43 \%$ de los menores de 2 años tuvo una combinación de telemedicina con consultas presenciales, para los mayores de 3 años fue entre $6 \%$ y $10 \%$. En este tramo etario, entre $71 \%$ y $74 \%$ de los niños no tuvo ninguna consulta presencial (ver tabla A12).
Por último, como se observa en la tabla A13, de aquellos niños que tenían algún estudio médico, procedimiento o tratamiento en curso o previsto durante la pandemia, el $76 \%$ no pudo realizar ninguno, mientras que $16 \%$ pudo realizarlos todos. Una vez más se encuentran diferencias significativas por edad: $50 \%$ de los menores de 2 años pudo completar todos los tratamientos versus $4 \%$ de los niños de 6 años o más. Cabe destacar que $61 \%$ de los niños tuvo que retrasar alguna vacuna prevista.

Respecto a los mayores de 65 años, grupo especialmente vulnerable por presentar un mayor riesgo asociado a la COVID-19, peor pronóstico debido a las comorbilidades, los síndromes geriátricos y la fragilidad asociada al envejecimiento ${ }^{(4)}$, destaca el menor acceso a consultas presenciales. El $90 \%$ no tuvo acceso a consulta presencial en domicilio y $42 \%$ en policlínica, lo cual puede ser consecuencia de la estrategia de protección y distanciamiento recomendada por la autoridad sanitaria.

En cuanto a la necesidad de consultas en policlínicas, tuvieron más necesidad los de Montevideo, los menos privados y los que declaran un buen estado de salud. Si nos detenemos en consultas a domicilio, declaran haber tenido mayor necesidad los que tienen educación primaria, un mal estado de salud, al menos una condición médica diagnosticada y cobertura a través de IAMC. Entre quienes tenían agendadas consultas en policlínica, pudieron concretar menos (en cualquier modalidad) las mujeres que los hombres, los que tienen cobertura a través de ASSE y aquellos con privación relativa. Si eran a domicilio, se mantiene lo encontrado por sexo, y se agrega que pudieron concretar menos los de Montevideo respecto al interior y los que no tenían condición médica diagnosticada.

\section{Discusión}

La ESAC, realizada a residentes en Uruguay de 18 años o más, muestra que las mujeres, los más educados y con menor privación relativa son los que declaran mayor necesidad de consultas en el período comprendido entre el 13 de marzo y el 30 de mayo de 2020. Sin embargo, solo se encuentra que tuvieron problemas de acceso las mujeres y quienes tienen cobertura pública de cualquier grupo etario.

En relación a los cambios que la pandemia tuvo sobre la modalidad de la práctica clínica, se observa la alta participación de la telemedicina. A su vez, el acceso a consultas presenciales estuvo más concentrado en los jóvenes y en los que tienen cobertura pública. En el caso de los adultos de 65 años y más, prácticamente no accedieron a consultas presenciales en domicilio, y un poco menos de la mitad no tuvo consultas presenciales en po- 
liclínica. La modalidad de telemedicina fue definida por el centro de salud en $80 \%$ de los casos cuando era en policlínica y en $56 \%$ cuando era en domicilio.

El 50\% de los entrevistados declara que el centro de salud le canceló o modificó la modalidad o la fecha de la consulta, destaca la alta satisfacción en cómo se gestionaron estos cambios. Sin embargo, se encuentran diferencias por sexo y cobertura, estando menos satisfechas las mujeres y los usuarios de ASSE.

Un aspecto importante a destacar es el alto porcentaje de estudios médicos, procedimientos o tratamientos en curso o previstos durante la pandemia, que debieron ser suspendidos o posponerse. Únicamente $11 \%$ de los entrevistados pudo realizar todos los estudios previstos o continuar con tratamientos en cursos. En este caso los usuarios de ASSE son quienes pudieron realizarlos en menor medida.

Centrándonos en los niños, la mitad necesitó al menos un control pediátrico habiendo accedido $70 \%$ de ellos, mayoritariamente de forma no presencial (60\%), y encontrando diferencias por edades. El acceso no presencial es mayor para los de 3 años o más y en el caso de los mayores de 5 años solo $57 \%$ de aquellos que necesitaron un control, pudo acceder. Destaca que $76 \%$ de los niños no pudo realizar ningún estudio médico, procedimiento o tratamiento en curso, y apenas el $4 \%$ de los de 6 años o más pudo completar todos los tratamientos. En cuanto a las vacunas, el $61 \%$ de los niños tuvo que retrasar alguna vacuna prevista.

Finalmente, encontramos diferencias significativas entre las instituciones, al considerar cinco IAMC de Montevideo en las cuales el número de encuestados fue superior a cien. El porcentaje de entrevistados que no tuvo ninguna consulta presencial varió entre $36 \%$ y $72 \%$ según la institución, mientras que quienes tuvieron todas las consultas presenciales varían entre $2 \%$ y $24 \%$. Del mismo modo, la satisfacción con la forma en que la IAMC gestionó los cambios e imprevistos surgidos en la atención a raíz de la crisis sanitaria, varió entre $42 \%$ y $64 \%$. A su vez, se encontraron diferencias entre algunas de estas instituciones y ASSE, presentando mayor satisfacción esta última, pudiendo esto estar asociada al mantenimiento de la presencialidad en las consultas de policlínica.

Hacia el futuro, y dado que el período de la crisis sanitaria y sus consecuencias durará más de lo que se podía prever inicialmente, surgen diversas preocupaciones que será necesario atender para garantizar los derechos de los usuarios y no profundizar problemas de acceso y/o brechas. En primer lugar, adecuar los tiempos de espera máximos establecidos por el MSP, garantizando el acceso en tiempos y modalidad adecuados. En este sentido, si bien la telemedicina (sincrónica, asincrónica, etc.) no es nueva, su uso generalizado ha sido una consecuencia de la pandemia. Las instituciones, que en general son las que definieron esta modalidad, deben realizar un ajuste a la forma de asignación y priorización de los pacientes (triaje) debiéndose considerar las características de los mismos.

\section{Abstract}

Introduction: the COVID-19 pandemic has drastically changed the outpatient medical care modalities and health care services in general. Since March 15, 2020, Uruguay has implemented changes in the health system aiming to focus the resources available and to avoid infection.

Objective: to present the results of the survey on health and access to medical care (ESAC) during the health emergency caused by the COVID-19 pandemic in Uruguay from March 13 to May 20, 2020. The study aims to present the impact of the pandemic on several aspects in connection with health care services.

Method: the ESAC survey, aimed at residents in Uruguay who are over 18 years old, consisted of a self-administered online questionnaire applied between 10 and 27 July, 2020. The total sample included 1750 surveys that assessed medical care and health services.

Results: the health crisis favored changes in the clinical practice modalities, what was evident in the significant increase of telemedicine, what was mainly defined by each medical institution. The survey revealed a high degree of satisfaction among users, in terms of response to the pandemic and the services offered by their institutions. However, it is worth pointing out that a high percentage of medical tests, procedures or ongoing or intended therapies were cancelled or postponed. In the case of children under 12 years old, over half of them received their vaccinations later than what was expected.

Conclusions: in the future, the health system must pay special attention to the way in which new consultation modalities are defined and implemented, as well as the maximum waiting times and the appropriate provision of services for chronic patients, to ensure access to timely and quality medical care.

\section{Resumo}

Introdução: a pandemia causada pela COVID 19 mudou dramaticamente as modalidades do atendimento médico ambulatorial e dos cuidados de saúde. O Uruguai, desde 15 de março de 2020, implementou uma série de mudanças no sistema de saúde com o objetivo de focar os recursos disponíveis e prevenir infecções.

Objetivo: apresentar os resultados da Pesquisa de Saúde e Acesso à Assistência Médica (ESAC) durante a emergência sanitária causada pela COVID-19 no Uru- 
guai de 13 de março a 30 de maio de 2020 . O objetivo é mostrar o impacto que a pandemia teve sobre alguns aspectos do atendimento médico recebido.

Material e métodos: a ESAC, destinado a residentes no Uruguai maiores de 18 anos, foi aplicada por meio de questionário autoaplicável na internet entre 10 e 27 de julho de 2020. A amostra total é de 1.750 inquéritos e informações coletadas relacionadas à saúde e aos cuidados médicos.

Resultados: a crise de saúde levou a uma mudança na modalidade de prática clínica com alta participação da telemedicina, que era definida em grande parte pela instituição de saúde. Durante a pandemia se registrou uma elevada satisfação na gestão e cuidados de saúde. No entanto, destaca-se o alto percentual de exames, procedimentos ou tratamentos médicos em andamento ou planejados que foram suspensos ou adiados. No caso de crianças menores de 12 anos, verifica-se que mais da metade teve que adiar uma vacinação planejada.

Conclusões: futuramente, será necessário que o sistema de saúde preste atenção especial à forma como são definidas e implementadas novas modalidades de consulta, tempos máximos de espera e o correto atendimento aos portadores de doenças crônicas, para garantir o acesso ao atendimento médico oportuno e de qualidade.

\section{Referencias bibliográficas}

1. Uruguay. Ministerio de Salud Pública. Junta Nacional de Salud. Resolución No 2: Medidas COVID-19. Montevideo, 2020.

2. Uruguay. Ministerio de Salud Pública. Cronología de acciones en relación a la situación de coronavirus COVID-19. Montevideo,2020. 11p.

3. Borooah VK. Logit and Probit: ordered and multinomial models. Thousand Oaks, CA: Sage, 2001.

4. Bonanad C, García-Bla S, Tarazona-Santabalbina FJ, Díez-Villanueva P, Ayesta A, Forés JS, et al. Coronavirus: la emergencia geriátrica de 2020. Documento conjunto de la Sección de Cardiología Geriátrica de la Sociedad Española de Cardiología y la Sociedad Española de Geriatría y Gerontología. Rev Esp Cardiol 2020; 73(7):569-76.

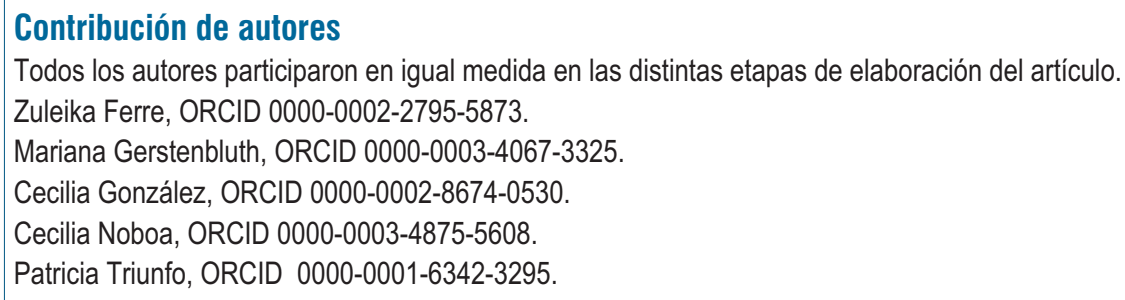




\section{Anexo 1. Tablas descriptivas}

Para todos los cuadros se hicieron pruebas de diferencias de media por subgrupos, resultados a disposición ante requerimiento.

Tabla A1. Porcentaje de consultas médicas necesitadas, concretadas y modalidad en la que se concretó por tipo de consulta.

\begin{tabular}{|c|c|c|c|c|c|c|c|c|}
\hline \multirow[t]{3}{*}{ Tipo de consulta } & \multicolumn{3}{|c|}{$\begin{array}{l}\text { Tenía agendado o tuvo necesidad de realizar } \\
\text { una consulta médica }\end{array}$} & \multicolumn{3}{|c|}{$\begin{array}{l}\text { Pudo concretar al menos una de esas consultas } \\
\text { (en cualquier modalidad) }\end{array}$} & \multicolumn{2}{|c|}{$\begin{array}{l}\text { Al menos una de esas } \\
\text { consultas fue: }\end{array}$} \\
\hline & & & & & & & \multirow{2}{*}{$\begin{array}{c}\text { Presencial } \\
\%\end{array}$} & \multirow{2}{*}{$\begin{array}{c}\text { Telemedicina } \\
\%\end{array}$} \\
\hline & $\%$ & Intervalo o & nza (95\%) & $\%$ & Intervalo & anza (95\%) & & \\
\hline De cualquier tipo & 47,5 & 37,9 & 57,1 & - & - & - & - & - \\
\hline $\begin{array}{l}\text { En policlínica o } \\
\text { consultorio }\end{array}$ & 33,7 & 26,2 & 41,2 & 77,3 & 69,5 & 85,2 & 54,3 & 73,4 \\
\hline $\begin{array}{l}\text { Médico de radio a } \\
\text { domicilio }\end{array}$ & 15,0 & 4,9 & 25,2 & 91,4 & 81,7 & 101,1 & 37,4 & 79,4 \\
\hline Emergencia móvil & 5,6 & 2,8 & 8,5 & 86,1 & 64,9 & 107,2 & 54,8 & 42,1 \\
\hline
\end{tabular}

Fuente: Elaboración propia en base a ESAC-2020 
Tabla A2. Porcentaje de personas que tenía agendado y accedió al menos a una consulta en policlínica según sexo, edad, nivel educativo, región, cobertura médica, estado de salud, condición médica diagnosticada y privación relativa.

\begin{tabular}{|c|c|c|c|c|c|c|}
\hline \multirow[b]{3}{*}{ Total } & \multicolumn{3}{|c|}{$\begin{array}{l}\text { Tenía agendada o tuvo necesidad de una consulta médica en } \\
\text { policlínica o consultorio }\end{array}$} & \multicolumn{3}{|c|}{ Accedió a una consulta médica (sin importar la modalidad) } \\
\hline & \multirow{2}{*}{$\begin{array}{c}\% \\
33,7\end{array}$} & \multicolumn{2}{|c|}{ Intervalo de confianza (95\%) } & \multirow{2}{*}{$\begin{array}{c}\% \\
77,33\end{array}$} & \multicolumn{2}{|c|}{ Intervalo de confianza (95\%) } \\
\hline & & 26,23 & 41,15 & & 69,46 & 85,20 \\
\hline \multicolumn{7}{|l|}{ Sexo } \\
\hline Femenino & 41,1 & 34,21 & 48,02 & 73,68 & 63,61 & 83,74 \\
\hline Masculino & 26,2 & 14,90 & 37,54 & 82,93 & 70,96 & 94,89 \\
\hline \multicolumn{7}{|l|}{ Edad } \\
\hline Menos de 30 & 29,6 & 12,90 & 46,20 & 90,69 & 84,26 & 97,12 \\
\hline 30 a 44 & 28,4 & 19,51 & 37,33 & 60,82 & 41,69 & 79,96 \\
\hline 45 a 54 & 31,4 & 19,38 & 43,49 & 80,96 & 69,99 & 91,93 \\
\hline 55 a 64 & 37,8 & 20,95 & 54,64 & 78,93 & 65,71 & 92,15 \\
\hline 65 y más & 35,8 & 14,78 & 56,81 & 78,56 & 58,72 & 98,40 \\
\hline \multicolumn{7}{|l|}{ Nivel educativo } \\
\hline Primaria & 23,0 & 6,33 & 39,70 & 72,64 & 45,34 & 99,94 \\
\hline Secundaria & 39,1 & 32,18 & 45,93 & 81,72 & 73,01 & 90,42 \\
\hline Terciaria & 37,7 & 34,56 & 40,93 & 70,85 & 66,17 & 75,53 \\
\hline \multicolumn{7}{|l|}{ Región } \\
\hline Interior & 33,7 & 22,16 & 45,27 & 78,66 & 67,31 & 90,00 \\
\hline Montevideo & 33,4 & 25,82 & 41,02 & 75,46 & 65,22 & 85,71 \\
\hline \multicolumn{7}{|l|}{ Cobertura médica } \\
\hline ASSE & 43,5 & 27,31 & 59,76 & 72,93 & 51,37 & 94,50 \\
\hline IAMC & 36,4 & 27,45 & 45,32 & 78,34 & 69,59 & 87,10 \\
\hline Seguros privados & 41,1 & 22,13 & 60,15 & 88,70 & 78,25 & 99,15 \\
\hline Otros & 7,8 & 0,69 & 14,85 & 69,54 & 50,35 & 88,74 \\
\hline \multicolumn{7}{|l|}{ Estado de salud } \\
\hline Malo o regular & 39,5 & 13,70 & 65,27 & 82,94 & 66,44 & 99,45 \\
\hline Bueno & 40,3 & 30,39 & 50,27 & 79,50 & 67,62 & 91,37 \\
\hline $\begin{array}{l}\text { Muy bueno o } \\
\text { excelente }\end{array}$ & 25,1 & 16,26 & 34,00 & 70,17 & 57,19 & 83,15 \\
\hline \multicolumn{7}{|c|}{ Condición médica diagnosticada } \\
\hline Ninguna & 24,4 & 15,15 & 33,37 & 69,48 & 54,62 & 84,33 \\
\hline Al menos una & 40,7 & 29,75 & 51,71 & 80,82 & 71,80 & 89,85 \\
\hline \multicolumn{7}{|l|}{ Privación relativa } \\
\hline No & 40,9 & 33,76 & 47,93 & 76,98 & 67,31 & 86,64 \\
\hline Sí & 26,2 & 15,13 & 37,34 & 77,89 & 64,51 & 91,26 \\
\hline
\end{tabular}

Fuente: Elaboración propia en base a ESAC-2020. 
Tabla A3. Porcentaje de consultas en cada modalidad (entre quienes tenían agendada o necesitaron consultas en policlínicas o domicilio y pudieron realizar al menos una consulta en el período)

\begin{tabular}{lrrr}
\hline & $\%$ & \multicolumn{2}{c}{ Intervalo de confianza (95\%) } \\
\hline Policlinica & & & 47,1 \\
Presencial & 38,1 & 29,1 & 68,2 \\
Telemedicina & 59,2 & 50,3 & 4,7 \\
Otras & 2,7 & 0,7 & \\
Total & 100,0 & & 52,3 \\
Domicilio & & & 98,0 \\
Presencial & 27,0 & 1,7 & 3,0 \\
Telemedicina & 71,7 & 45,4 & \\
Otras & 1,3 & 0,0 & \\
& 100,0 & & \\
\hline
\end{tabular}

Fuente: Elaboración propia en base a ESAC-2020. 
Tabla A4. Proporción de consultas presenciales en policlínica sobre el total de consultas para el total y por subgrupos.

\begin{tabular}{|c|c|c|c|}
\hline & $\%$ & \multicolumn{2}{|c|}{ Intervalo de confianza (95\%) } \\
\hline \multicolumn{4}{|l|}{ Total } \\
\hline Sin consultas presenciales & 44,0 & 33,4 & 55,0 \\
\hline Combinación de presencial y otra & 32,4 & 22,4 & 44,4 \\
\hline Todas las consultas presenciales & 23,6 & 15,1 & 34,9 \\
\hline \multicolumn{4}{|l|}{ Sexo } \\
\hline \multicolumn{4}{|l|}{ Sin consultas presenciales } \\
\hline Femenino & 41,4 & 31,0 & 52,8 \\
\hline Masculino & 47,1 & 28,1 & 67,0 \\
\hline \multicolumn{4}{|l|}{ Combinación de presencial y otra } \\
\hline Femenino & 33,1 & 23,7 & 44,0 \\
\hline Masculino & 31,7 & 14,5 & 55,8 \\
\hline Todas las consultas presenciales & & & 0 \\
\hline Femenino & 25,5 & 14,6 & 40,6 \\
\hline Masculino & 21,2 & 10,0 & 39,5 \\
\hline \multicolumn{4}{|l|}{ Edad } \\
\hline \multicolumn{4}{|l|}{ Sin consultas presenciales } \\
\hline Menos de 30 & 24,7 & 10,8 & 47,0 \\
\hline 30 a 44 & 50,9 & 31,9 & 69,7 \\
\hline 45 a 54 & 62,5 & 37,2 & 82,4 \\
\hline 55 a 64 & 40,0 & 22,9 & 60,0 \\
\hline 65 y más & 41,8 & 21,7 & 65,2 \\
\hline \multicolumn{4}{|l|}{ Combinación de presencial y otra } \\
\hline Menos de 30 & 15,3 & 6,6 & 31,6 \\
\hline 30 a 44 & 29,8 & 17,7 & 45,5 \\
\hline 45 a 54 & 29,0 & 11,5 & 56,2 \\
\hline 55 a 64 & 25,2 & 12,6 & 44,0 \\
\hline 65 y más & 52,9 & 29,4 & 75,2 \\
\hline Todas las consultas presenciales & 0,0 & 0,0 & 0,0 \\
\hline Menos de 30 & 60,0 & 33,0 & 82,0 \\
\hline 30 a 44 & 19,3 & 10,9 & 31,9 \\
\hline 45 a 54 & 8,5 & 3,3 & 20,0 \\
\hline 55 a 64 & 34,8 & 17,8 & 56,8 \\
\hline 65 y más & 5,2 & 1,6 & 15,6 \\
\hline
\end{tabular}

Nivel educativo

Sin consultas presenciales

Primaria

Secundaria

Terciaria
14,7

53,4

42,8
1,9

40,5

36,8
60,3

65,9

48,9 
Tabla A4. viene de la página anterior

\begin{tabular}{|c|c|c|c|}
\hline \multirow[b]{2}{*}{ Combinación de presencial y otra } & & \multicolumn{2}{|c|}{ Intervalo de confianza (95\%) } \\
\hline & & 0,0 & 0,0 \\
\hline Primaria & 42,9 & 11,4 & 81,5 \\
\hline Secundaria & 28,1 & 18,6 & 40,2 \\
\hline Terciaria & 35,6 & 30,0 & 41,6 \\
\hline Todas las consultas presenciales & 0,0 & 0,0 & 0,0 \\
\hline Primaria & 42,4 & 12,3 & 79,4 \\
\hline Secundaria & 18,4 & 10,1 & 31,2 \\
\hline Terciaria & 21,7 & 16,9 & 27,3 \\
\hline \multicolumn{4}{|l|}{ Región } \\
\hline \multicolumn{4}{|l|}{ Sin consultas presenciales } \\
\hline Interior & 45,5 & 31,0 & 60,8 \\
\hline Montevideo & 41,7 & 28,0 & 56,9 \\
\hline \multicolumn{4}{|l|}{ Combinación de presencial y otra } \\
\hline Interior & 27,2 & 16,1 & 42,1 \\
\hline Montevideo & 39,8 & 24,2 & 57,9 \\
\hline \multicolumn{4}{|l|}{ Todas las consultas presenciales } \\
\hline Interior & 27,3 & 14,7 & 45,0 \\
\hline Montevideo & 18,4 & 11,5 & 28,2 \\
\hline \multicolumn{4}{|l|}{ Cobertura médica } \\
\hline \multicolumn{4}{|l|}{ Sin consultas presenciales } \\
\hline ASSE & 13,3 & 6,0 & 27,1 \\
\hline IAMC & 51,9 & 38,7 & 64,8 \\
\hline Seguros privados & 65,7 & 33,9 & 87,7 \\
\hline Otros & 39,1 & 17,1 & 66,6 \\
\hline \multicolumn{4}{|l|}{ Combinación de presencial y otra } \\
\hline ASSE & 29,7 & 12,1 & 56,6 \\
\hline IAMC & 34,0 & 21,8 & 48,7 \\
\hline Seguros privados & 20,5 & 7,5 & 45,0 \\
\hline Otros & 38,5 & 14,3 & 70,2 \\
\hline \multicolumn{4}{|l|}{ Todas las consultas presenciales } \\
\hline ASSE & 56,9 & 31,6 & 79,1 \\
\hline IAMC & 14,2 & 8,1 & 23,5 \\
\hline Seguros privados & 13,8 & 4,3 & 36,1 \\
\hline Otros & 22,4 & 7,2 & 51,8 \\
\hline \multicolumn{4}{|l|}{ Estado de salud } \\
\hline \multicolumn{4}{|l|}{ Sin consultas presenciales } \\
\hline Malo o regular & 38,8 & 18,1 & 64,6 \\
\hline Bueno & 44,9 & 29,0 & 61,9 \\
\hline Muy bueno o excelente & 46,4 & 31,5 & 62,0 \\
\hline
\end{tabular}


Tabla A4. viene de la página anterior

\begin{tabular}{|c|c|c|c|}
\hline & $\%$ & \multicolumn{2}{|c|}{ Intervalo de confianza (95\%) } \\
\hline \multicolumn{4}{|c|}{ Combinación de presencial y otra } \\
\hline Malo o regular & 41,9 & 20,5 & 66,9 \\
\hline Bueno & 35,6 & 19,9 & 55,1 \\
\hline Muy bueno o excelente & 20,1 & 12,8 & 30,2 \\
\hline \multicolumn{4}{|c|}{ Todas las consultas presenciales } \\
\hline Malo o regular & 19,3 & 5,7 & 48,4 \\
\hline Bueno & 19,6 & 9,3 & 36,7 \\
\hline Muy bueno o excelente & 33,5 & 18,5 & 52,7 \\
\hline \multicolumn{4}{|c|}{ Condición médica diagnosticada } \\
\hline \multicolumn{4}{|l|}{ Sin consultas presenciales } \\
\hline Ninguna & 48,8 & 31,2 & 66,6 \\
\hline Al menos una & 42,0 & 29,6 & 55,6 \\
\hline \multicolumn{4}{|c|}{ Combinación de presencial y otra } \\
\hline Ninguna & 17,1 & 10,4 & 26,8 \\
\hline Al menos una & 38,5 & 25,5 & 53,3 \\
\hline \multicolumn{4}{|c|}{ Todas las consultas presenciales } \\
\hline Ninguna & 34,1 & 17,6 & 55,6 \\
\hline Al menos una & 19,4 & 10,7 & 32,7 \\
\hline \multicolumn{4}{|l|}{ Privación relativa } \\
\hline \multicolumn{4}{|l|}{ Sin consultas presenciales } \\
\hline No & 48,9 & 35,3 & 62,6 \\
\hline Sí & 35,7 & 20,7 & 54,2 \\
\hline \multicolumn{4}{|c|}{ Combinación de presencial y otra } \\
\hline No & 30,5 & 18,1 & 46,7 \\
\hline Sí & 35,6 & 20,6 & 54,1 \\
\hline \multicolumn{4}{|c|}{ Todas las consultas presenciales } \\
\hline No & 20,6 & 12,1 & 32,8 \\
\hline Sí & 28,7 & 13,4 & 51,0 \\
\hline
\end{tabular}

Fuente: elaboración propia en base a ESAC-2020. 
Tabla A5. Proporción de consultas presenciales de médico de radio o especialista a domicilio sobre el total de consultas para el total y por subgrupos.

\begin{tabular}{|c|c|c|c|}
\hline & $\%$ & \multicolumn{2}{|c|}{ Intervalo de confianza (95\%) } \\
\hline \multicolumn{4}{|l|}{ Total } \\
\hline Sin consultas presenciales & 60,2 & 24,7 & 87,5 \\
\hline Combinación de presencial y otra & 24,5 & 8,0 & 54,8 \\
\hline Todas las consultas presenciales & 15,3 & 4,8 & 39,5 \\
\hline \multicolumn{4}{|l|}{ Sexo } \\
\hline \multicolumn{4}{|l|}{ Sin consultas presenciales } \\
\hline Femenino & 26,7 & 15,9 & 41,3 \\
\hline Masculino & 84,0 & 40,6 & 97,6 \\
\hline \multicolumn{4}{|l|}{ Combinación de presencial y otra } \\
\hline Femenino & 41,5 & 23,4 & 62,2 \\
\hline Masculino & 12,4 & 1,7 & 54,3 \\
\hline \multicolumn{4}{|l|}{ Todas las consultas presenciales } \\
\hline Femenino & 31,8 & 15,1 & 54,9 \\
\hline Masculino & 3,5 & 0,7 & 16,9 \\
\hline \multicolumn{4}{|l|}{ Edad } \\
\hline \multicolumn{4}{|l|}{ Sin consultas presenciales } \\
\hline Menos de 30 & 22,4 & 8,2 & 48,1 \\
\hline 30 a 44 & 8,7 & 2,5 & 26,0 \\
\hline 45 a 54 & 9,1 & 2,7 & 26,8 \\
\hline 55 a 64 & 65,7 & 39,4 & 84,9 \\
\hline 65 y más & 90,2 & 56,7 & 98,5 \\
\hline \multicolumn{4}{|l|}{ Combinación de presencial y otra } \\
\hline Menos de 30 & 46,9 & 17,9 & 78,1 \\
\hline 30 a 44 & 69,8 & 35,6 & 90,6 \\
\hline 45 a 54 & 46,2 & 12,9 & 83,2 \\
\hline 55 a 64 & 24,4 & 10,4 & 47,4 \\
\hline 65 y más & 5,0 & 0,8 & 27,0 \\
\hline \multicolumn{4}{|l|}{ Todas las consultas presenciales } \\
\hline Menos de 30 & 30,7 & 10,6 & 62,5 \\
\hline 30 a 44 & 21,5 & 6,5 & 51,9 \\
\hline 45 a 54 & 44,6 & 11,5 & 83,3 \\
\hline 55 a 64 & 9,9 & 3,5 & 25,3 \\
\hline 65 y más & 4,7 & 0,7 & 26,4 \\
\hline
\end{tabular}


Tabla A5. Viene de la página anterior

$$
\% \quad \text { Intervalo de confianza (95\%) }
$$

\section{Nivel educativo}

Sin consultas presenciales

Primaria

80,2

26,4

97,9

Secundaria

37,3

19,5

59,4

Terciaria

31,8

22,3

43,2

Combinación de presencial y otra

Primaria

9,9

0,8

61,1

Secundaria

47,3

27,9

67,6

Terciaria

25,7

16,3

38,1

Todas las consultas presenciales

Primaria

9,9

0,8

61,1

Secundaria

15,4

7,6

28,8

Terciaria

42,5

31,2

54,6

\section{Región}

Sin consultas presenciales

Interior

Montevideo

Combinación de presencial y otra

Interior

Montevideo

Todas las consultas presenciales

Interior

Montevideo

\section{Cobertura médica}

Sin consultas presenciales

ASSE

IAMC

Seguros privados

Otros

Combinación de presencial y otra

ASSE

IAMC

Seguros privados

Otros
78,1

20,8

16,2

42,8

5,7

36,5

50,6

63,3

34,8

5,1

32,8

21,7

37,2

81,8
38,0

95,4

10,9

36,0

3,3

52,6

20,5

68,4

1,3

22,1

15,6

64,0 
Tabla A5. Viene de la página anterior

\begin{tabular}{|c|c|c|c|}
\hline & $\%$ & \multicolumn{2}{|c|}{ Intervalo de confianza (95\%) } \\
\hline \multicolumn{4}{|c|}{ Todas las consultas presenciales } \\
\hline ASSE & 16,6 & 2,9 & 57,0 \\
\hline IAMC & 15,0 & 4,1 & 42,5 \\
\hline Seguros privados & 28,0 & 9,0 & 60,5 \\
\hline Otros & 13,2 & 3,0 & 42,6 \\
\hline \multicolumn{4}{|l|}{ Estado de salud } \\
\hline \multicolumn{4}{|l|}{ Sin consultas presenciales } \\
\hline Malo o regular & 78,9 & 32,1 & 96,7 \\
\hline Bueno & 31,7 & 12,7 & 59,6 \\
\hline Muy bueno o excelente & 36,4 & 19,3 & 57,9 \\
\hline \multicolumn{4}{|c|}{ Combinación de presencial y otra } \\
\hline Malo o regular & 7,5 & 1,4 & 31,3 \\
\hline Bueno & 50,1 & 25,0 & 75,2 \\
\hline Muy bueno o excelente & 47,4 & 26,5 & 69,2 \\
\hline \multicolumn{4}{|c|}{ Todas las consultas presenciales } \\
\hline Malo o regular & 13,6 & 1,8 & 58,0 \\
\hline Bueno & 18,2 & 8,8 & 33,9 \\
\hline Muy bueno o excelente & 16,2 & 8,1 & 29,9 \\
\hline \multicolumn{4}{|c|}{ Condición médica diagnosticada } \\
\hline \multicolumn{4}{|l|}{ Sin consultas presenciales } \\
\hline Ninguna & 25,5 & 8,8 & 54,7 \\
\hline Al menos una & 65,4 & 27,3 & 90,5 \\
\hline \multicolumn{4}{|c|}{ Combinación de presencial y otra } \\
\hline Ninguna & 59,5 & 25,2 & 86,5 \\
\hline Al menos una & 19,2 & 5,6 & 49,0 \\
\hline \multicolumn{4}{|c|}{ Todas las consultas presenciales } \\
\hline Ninguna & 15,0 & 5,0 & 37,0 \\
\hline Al menos una & 15,3 & 4,0 & 44,0 \\
\hline \multicolumn{4}{|l|}{ Privación relativa } \\
\hline \multicolumn{4}{|l|}{ Sin consultas presenciales } \\
\hline No & 24,8 & 13,9 & 40,1 \\
\hline Sí & 74,8 & 32,0 & 94,9 \\
\hline \multicolumn{4}{|c|}{ Combinación de presencial y otra } \\
\hline No & 51,5 & 31,6 & 71,0 \\
\hline Sí & 13,4 & 2,6 & 47,0 \\
\hline \multicolumn{4}{|c|}{ Todas las consultas presenciales } \\
\hline No & 23,7 & 13,3 & 38,6 \\
\hline Sí & 11,8 & 1,9 & 47,8 \\
\hline
\end{tabular}

Fuente: Elaboración propia en base a ESAC-2020. 
Tabla A6. Porcentaje de entrevistados que pudieron realizar consultas médicas según quien definió la modalidad de consulta

\begin{tabular}{lcc}
\hline \multicolumn{1}{c}{ Quién definió } & $\begin{array}{c}\text { En consultas agendadas en consultorio } \\
\text { (policlínica) (\%) }\end{array}$ & $\begin{array}{c}\text { En consultas agendadas a domicilio (médico de } \\
\text { radio) }\end{array}$ \\
\hline Presencial & & 56,9 \\
El centro de salud & 52,5 & 38,5 \\
El entrevistado & 44,3 & 4,6 \\
No sabe & 3,2 & 25,6 \\
Telefónicas o por video llamada & & 72,1 \\
El centro de salud & 79,5 & 2,3 \\
El entrevistado & 19,9 & 2,3 \\
No sabe & 0,7 & \\
\hline
\end{tabular}

Fuente: elaboración propia en base a ESAC-2020.

Tabla A7. Porcentaje de entrevistados que hubiesen preferido tener la consulta en otra modalidad según tipo de consulta.

\begin{tabular}{lc}
\hline Consultas agendadas en consultorio & $\%$ \\
Modalidad presencial & 4,8 \\
Modalidad telefónica & 35,7 \\
Consultas agendadas a con médico de radio o especialista a domicilio & 1,7 \\
Modalidad presencial & 19,2 \\
Modalidad telefónica & \\
\hline
\end{tabular}

Fuente: elaboración propia en base a ESAC-2020.

Tabla A8. Motivos del cambio en la modalidad de la consulta, cancelación o cambio de fecha.

\begin{tabular}{lc}
\hline Motivos & $\%$ \\
\hline El centro de salud no me dio otra opción & 49,9 \\
Temor a salir & 2,3 \\
Temor a asistir a un centro de salud & 9,7 \\
Temor a usar el trasporte público & 3,8 \\
Mi problema no tenía relevancia por lo que preferí no cargar al equipo de salud & 27,9 \\
Otros motivos no relacionados con la emergencia sanitaria & 6,3 \\
Total & 100,0 \\
\hline
\end{tabular}

Fuente: elaboración propia en base a ESAC-2020. 
Tabla A9. Porcentaje de estudios médicos, procedimientos o tratamientos en curso o previstos realizados por subgrupos.

\begin{tabular}{|c|c|c|c|}
\hline \multirow[b]{2}{*}{ Realizó todos } & \multirow{2}{*}{$\begin{array}{c}\% \\
10,7\end{array}$} & \multicolumn{2}{|c|}{ Intervalo de confianza (95\%) } \\
\hline & & 6,8 & 16,4 \\
\hline Realizó algunos & 17,3 & 10,7 & 26,8 \\
\hline Ninguno estudio & 72,0 & 60,4 & 81,2 \\
\hline \multicolumn{4}{|l|}{ Realizó todos los controles } \\
\hline \multicolumn{4}{|l|}{ Sexo } \\
\hline Femenino & 10,0 & 6,8 & 13,0 \\
\hline Masculino & 11,6 & 2,0 & 21,2 \\
\hline \multicolumn{4}{|l|}{ Edad } \\
\hline Menos de 30 & 8,3 & 2,1 & 14,6 \\
\hline 30 a 44 & 10,7 & 5,2 & 16,3 \\
\hline 45 a 54 & 8,2 & 2,8 & 13,7 \\
\hline 55 a 64 & 12,8 & 2,0 & 23,6 \\
\hline 65 y más & 9,5 & 0,8 & 18,2 \\
\hline \multicolumn{4}{|l|}{ Nivel educativo } \\
\hline Primaria & - & - & - \\
\hline Secundaria & 17,1 & 9,4 & 24,7 \\
\hline Terciaria & 24,6 & 20,2 & 29,1 \\
\hline \multicolumn{4}{|l|}{ Región } \\
\hline Interior & 8,5 & 2,4 & 14,5 \\
\hline Montevideo & 14,8 & 9,6 & 20,1 \\
\hline \multicolumn{4}{|l|}{ Cobertura médica } \\
\hline ASSE & 5,9 & 0,6 & 11,2 \\
\hline IAMC & 13,9 & 6,7 & 21,1 \\
\hline Seguros privados & 29,7 & 7,5 & 51,9 \\
\hline Otros & 1,5 & 0,0 & 3,5 \\
\hline \multicolumn{4}{|l|}{ Estado de salud } \\
\hline Malo o regular & 5,0 & 0,0 & 10,2 \\
\hline Bueno & 14,6 & 7,1 & 22,1 \\
\hline Muy bueno o excelente & 11,4 & 3,0 & 19,7 \\
\hline \multicolumn{4}{|c|}{ Condición médica diagnosticada } \\
\hline Ninguna & 12,5 & 3,0 & 21,9 \\
\hline Al menos una & 9,5 & 4,8 & 14,2 \\
\hline \multicolumn{4}{|l|}{ Privación relativa } \\
\hline No & 18,6 & 11,6 & 25,7 \\
\hline Sí & 3,5 & 0,0 & 6,2 \\
\hline
\end{tabular}

Fuente: elaboración propia en base a ESAC-2020. 
Tabla A10. Satisfacción con la modalidad y atención médica recibida, porcentaje de personas de acuerdo o muy de acuerdo.

\begin{tabular}{|c|c|c|c|c|c|c|c|c|c|}
\hline & \multicolumn{3}{|c|}{$\begin{array}{l}\text { "La modalidad en la que tuve las consultas } \\
\text { fue la mejor para lo que yo necesitaba" }\end{array}$} & \multicolumn{3}{|c|}{$\begin{array}{c}\text { "La atención médica estuvo de acuerdo } \\
\text { a mis expectativas" }\end{array}$} & \multicolumn{3}{|c|}{$\begin{array}{l}\text { "En mi caso, el centro de salud al que } \\
\text { pertenezco gestionó correctamente todos los } \\
\text { cambios e imprevistos surgidos en la atención } \\
\text { en la crisis sanitaria" }\end{array}$} \\
\hline & $\%$ & Intervalo d & ianza (95\%) & $\%$ & Intervalo o & ianza (95\%) & $\%$ & Intervalo & inza (95\%) \\
\hline Total & 67,5 & & & 77,8 & & & 72,4 & & \\
\hline \multicolumn{10}{|l|}{ Sexo } \\
\hline Femenino & 65,7 & 55,1 & 75,0 & 71,8 & 61,9 & 79,9 & 71,0 & 44,3 & 88,3 \\
\hline Masculino & 69,5 & 43,2 & 87,2 & 84,1 & 66,0 & 93,5 & 73,6 & 65,0 & 80,7 \\
\hline \multicolumn{10}{|l|}{ Edad } \\
\hline Menos de 30 & 67,2 & 44,5 & 84,0 & 74,3 & 53,3 & 88,0 & 72,6 & 50,7 & 87,2 \\
\hline 30 a 44 & 66,5 & 46,5 & 81,9 & 79,9 & 68,5 & 88,0 & 78,4 & 67,1 & 86,6 \\
\hline 45 a 54 & 49,9 & 29,5 & 70,4 & 52,7 & 31,8 & 72,8 & 50,6 & 30,2 & 70,9 \\
\hline 55 a 64 & 74,6 & 58,5 & 85,9 & 76,7 & 60,9 & 87,4 & 73,0 & 56,7 & 84,8 \\
\hline 65 y más & 68,8 & 35,9 & 89,6 & 88,1 & 67,5 & 96,4 & 77,7 & 45,7 & 93,5 \\
\hline \multicolumn{10}{|l|}{ Nivel educativo } \\
\hline Primaria & 74,1 & 37,0 & 93,3 & 89,0 & 58,3 & 97,9 & 83,4 & 44,4 & 97,0 \\
\hline Secundaria & 64,8 & 52,9 & 74,9 & 74,7 & 63,9 & 83,1 & 67,9 & 56,4 & 77,6 \\
\hline Terciaria & 63,1 & 58,0 & 67,9 & 66,7 & 61,7 & 71,4 & 64,7 & 59,6 & 69,4 \\
\hline \multicolumn{10}{|l|}{ Región } \\
\hline Interior & 71,5 & 52,7 & 84,9 & 80,3 & 64,6 & 90,1 & 79,8 & 64,1 & 89,8 \\
\hline Montevideo & 60,7 & 45,9 & 73,8 & 73,7 & 62,7 & 82,4 & 59,9 & 45,2 & 73,0 \\
\hline \multicolumn{10}{|c|}{ Cobertura médica } \\
\hline ASSE & 69,0 & 44,5 & 86,0 & 80,2 & 55,6 & 92,9 & 87,2 & 75,0 & 93,9 \\
\hline IAMC & 68,5 & 51,2 & 81,8 & 77,3 & 63,9 & 86,7 & 68,2 & 50,6 & 81,8 \\
\hline $\begin{array}{l}\text { Seguros } \\
\text { privados }\end{array}$ & 55,1 & 22,1 & 84,1 & 88,8 & 75,5 & 95,3 & 87,0 & 71,1 & 94,8 \\
\hline Otros & 52,2 & 29,3 & 74,3 & 59,1 & 34,0 & 80,2 & 63,7 & 38,1 & 83,3 \\
\hline \multicolumn{10}{|l|}{ Estado de salud } \\
\hline Malo o regular & 73,6 & 43,2 & 91,1 & 79,0 & 51,9 & 92,9 & 83,5 & 60,1 & 94,5 \\
\hline Bueno & 62,0 & 45,5 & 76,1 & 82,2 & 72,1 & 89,2 & 66,0 & 48,8 & 79,8 \\
\hline $\begin{array}{l}\text { Muy bueno } 0 \\
\text { excelente }\end{array}$ & 67,9 & 53,2 & 79,7 & 69,2 & 54,3 & 80,9 & 66,2 & 51,5 & 78,4 \\
\hline \multicolumn{10}{|c|}{ Condición médica diagnosticada } \\
\hline Ninguna & 69,3 & 53,8 & 81,43 & 70,5 & 54,9 & 82,4 & 68,6 & 53,1 & 80,8 \\
\hline Al menos una & 66,9 & 49,0 & 80,97 & 80,4 & 67,6 & 89,0 & 73,8 & 57,0 & 85,6 \\
\hline \multicolumn{10}{|c|}{ Privación relativa } \\
\hline No & 60,8 & 47,7 & 72,46 & 75,5 & 65,2 & 83,6 & 64,1 & 50,8 & 75,5 \\
\hline Sí & 74,8 & 52,8 & 88,77 & 80,3 & 60,83 & 91,4 & 81,9 & 63,7 & 92,1 \\
\hline
\end{tabular}

Fuente: Elaboración propia en base a ESAC-2020. 
Tabla A11. Porcentaje de consultas pediátricas necesitadas, concretadas y modalidad en la que se concretó para menores de 12 años.

\begin{tabular}{|c|c|c|c|c|c|c|c|c|}
\hline & \multicolumn{3}{|c|}{$\begin{array}{c}\text { Tenía agendado o tuvo necesidad de realizar } \\
\text { una consulta médica }\end{array}$} & \multicolumn{3}{|c|}{$\begin{array}{l}\text { Pudo concretar al menos una de esas consultas } \\
\text { (en cualquier modalidad) }\end{array}$} & \multicolumn{2}{|c|}{$\begin{array}{l}\text { Al menos una de esas } \\
\text { consultas fue }\end{array}$} \\
\hline & & & & & & & Presencial & Telemedicina \\
\hline & $\%$ & \multicolumn{2}{|c|}{ Intervalo de confianza (95\%) } & \multirow{2}{*}{$\begin{array}{r}\% \\
70,2\end{array}$} & \multicolumn{2}{|c|}{ Intervalo de confianza (95\%) } & $\%$ & $\%$ \\
\hline Total & 51,9 & 40,3 & 63,4 & & 55,8 & 84,7 & 39,2 & 60,8 \\
\hline \multicolumn{9}{|l|}{ Región } \\
\hline Interior & 49,1 & 29,9 & 68,3 & 64,2 & 39,0 & 89,4 & & \\
\hline Montevideo & 55,0 & 43,7 & 66,3 & 76,2 & 63,5 & 88,9 & & \\
\hline \multicolumn{9}{|l|}{ Cobertura de salud } \\
\hline ASSE & - & - & - & - & - & - & & \\
\hline IAMC & 54,8 & 43,0 & 66,6 & 80,4 & 70,7 & 90,0 & & \\
\hline Seguros privados & 51,5 & 36,9 & 66,0 & 75,6 & 54,3 & 97,0 & & \\
\hline Otros & - & - & - & - & - & - & & \\
\hline \multicolumn{9}{|l|}{ Edad del niño } \\
\hline Hasta 2 años & 85,4 & 77,6 & 93,2 & 85,1 & 70,9 & 99,3 & & \\
\hline Entre 3 y 5 años & 47,6 & 26,2 & 69,0 & 80,4 & 64,7 & 96,1 & & \\
\hline 6 años o más & 44,6 & 27,6 & 61,7 & 57,3 & 31,4 & 83,3 & & \\
\hline
\end{tabular}

Fuente: Elaboración propia en base a ESAC-2020. 
Tabla A12. Proporción de consultas presenciales sobre el total de consultas de cada niño según región, cobertura de salud y edad.

\begin{tabular}{|c|c|c|c|}
\hline & $\%$ & \multicolumn{2}{|c|}{ Intervalo de confianza (95\%) } \\
\hline Sin consultas presenciales & 59,5 & 42,0 & 75,0 \\
\hline Combinación de presencial y otra & 19,3 & 11,1 & 31,5 \\
\hline Todas las consultas presenciales & 21,1 & 11,7 & 35,2 \\
\hline \multicolumn{4}{|l|}{ Región } \\
\hline \multicolumn{4}{|l|}{ Sin consultas presenciales } \\
\hline Interior & 70,0 & 45,2 & 86,8 \\
\hline Montevideo & 50,7 & 28,3 & 72,8 \\
\hline \multicolumn{4}{|l|}{ Combinación de presencial y otra } \\
\hline Interior & 17,2 & 6,9 & 37,0 \\
\hline Montevideo & 21,1 & 10,5 & 37,9 \\
\hline \multicolumn{4}{|l|}{ Todas las consultas presenciales } \\
\hline Interior & 12,8 & 4,7 & 30,3 \\
\hline Montevideo & 28,2 & 13,8 & 49,1 \\
\hline \multicolumn{4}{|l|}{ Cobertura de salud } \\
\hline \multicolumn{4}{|l|}{ Sin consultas presenciales } \\
\hline ASSE & - & - & - \\
\hline IAMC & 57,3 & 37,9 & 74,6 \\
\hline Seguros privados & 38,5 & 21,7 & 58,6 \\
\hline Otros & 30,6 & 4,0 & 82,3 \\
\hline \multicolumn{4}{|l|}{ Combinación de presencial y otra } \\
\hline ASSE & - & - & - \\
\hline IAMC & 20,7 & 11,2 & 35,1 \\
\hline Seguros privados & 21,9 & 10,5 & 40,1 \\
\hline Otros & 63,0 & 16,2 & 93,8 \\
\hline \multicolumn{4}{|l|}{ Todas las consultas presenciales } \\
\hline ASSE & - & - & - \\
\hline IAMC & 22,1 & 11,3 & 38,8 \\
\hline Seguros privados & 39,6 & 22,0 & 60,2 \\
\hline Otros & 6,4 & 0,7 & 40,2 \\
\hline \multicolumn{4}{|l|}{ Edad del niño } \\
\hline \multicolumn{4}{|l|}{ Sin consultas presenciales } \\
\hline Hasta 2 años & 31,7 & 16,6 & 52,1 \\
\hline Entre 3 y 5 años & 70,9 & 37,8 & 90,7 \\
\hline 6 años o más & 73,6 & 46,1 & 90,1 \\
\hline \multicolumn{4}{|l|}{ Combinación de presencial y otra } \\
\hline Hasta 2 años & 43,2 & 26,1 & 62,1 \\
\hline Entre 3 y 5 años & 6,1 & 1,7 & 19,4 \\
\hline 6 años o más & 9,6 & 3,1 & 25,9 \\
\hline \multicolumn{4}{|l|}{ Todas las consultas presenciales } \\
\hline Hasta 2 años & 25,0 & 12,1 & 44,8 \\
\hline Entre 3 y 5 años & 23,0 & 6,6 & 55,9 \\
\hline 6 años o más & 16,8 & 5,3 & 42,0 \\
\hline
\end{tabular}

Fuente: elaboración propia en base a ESAC-2020. 
Tabla A13. Estudios médicos, procedimientos o tratamientos en curso o previstos realizados para menores de 12 años según edad del niño, y vacunas obligatorias retrasadas de aquellos que tenían vacunas previstas.

\begin{tabular}{lccc} 
& $\%$ & \multicolumn{2}{c}{ Intervalo de confianza (95\%) } \\
\hline Realizó todos & 16,2 & 4,8 & 27,6 \\
Realizó algunos & 7,5 & 3,5 & 15,1 \\
Ningún estudio & 76,3 & 60,1 & 87,4 \\
Edad del niño (realizó todos los estudios) & & & \\
Hasta 2 años & 50,6 & 17,9 & 83,3 \\
Entre 3 y 5 años & 41,1 & 9,7 & 72,5 \\
6 años o más & 3,6 & 0,0 & 7,7 \\
Vacunas atrasadas & 60,9 & 42,9 & 78,9 \\
\hline
\end{tabular}

Fuente: elaboración propia en base a ESAC-2020. 